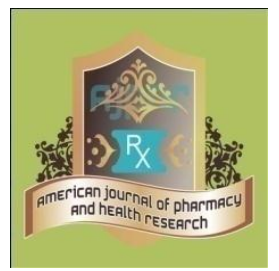

Research Article
AMERICAN JOURNAL OF PHARMACY AND HEALTH RESEARCH
www.ajphr.com

2018, Volume 6, Issue 09

ISSN: 2321-3647(online)

\title{
Clostridium Difficile Infection: in Human Diagnosis and Management
}

\author{
Krushnarao Bhorgir*, Kuldip Dhumal, Rohit Palve \\ Loknete Shree Dadapatil Pharate College of Pharmacy, Mandavgan Pharata, Pune. \\ Maharashtra, India 412211.
}

\begin{abstract}
Clostridium Difficile $(C D)$ is the most important cause of nosocomial diarrhea in adults. Illness may range from mild watery diarrhoea to life-threatening colitis. CD has now also emerged in the community in populations previously considered low risk. Emerging risk factors and disease recurrence represent continued challenges in the management of CDI. Diagnosis is based primarily on the detection of $C$. difficile toxin A or toxin B. These toxins primarily disrupt the cytoskeletal structure and the tight junctions of target cells causing cell rounding and ultimately cell death. The toxins trigger a complex cascade of host cellular responses to cause diarrhoea, inflammation and tissue necrosis- the major symptoms of CDI. The factors responsible for the epidemic of some C. difficile strains are poorly understood the organism has evolved over the last 8 years to become more virulent and resistant to antimicrobials causing a more severe form of the disease that has increased mortality and healthcare costs. The guideline provides recommendations for the diagnosis and management of patients with CDI as well as for the prevention and control of outbreaks while supplementing previously published guidelines. New molecular diagnostic stool tests will likely replace current enzyme immunoassay tests. We suggest treatment of patients be stratified depending on whether they have mild-to-moderate, severe or complicated disease. This chapter highlights the current knowledge on $C$. difficile how to produce infection in human, transmission, guidelines, Epidemiology, risk and diagnosis.
\end{abstract}

Keywords: Clostridium difficile, pathogenesis, guidelines, Epidemiology, risk factors, diagnosis. 


\section{INTRODUCTION}

Notable outbreaks Clostridium difficile was first isolated from the stool of a healthy infant by Hall and O'Toole in 1935. ${ }^{[1]}$ The species name was chosen to reflect the difficulty with its culture and isolation. Pseudo membranous colitis was first described in 1893. ${ }^{[6]}$ It was not until 1978, however that George and colleagues associated $C$. difficile with human disease and discovered that $C$. difficile was the organism responsible for the majority of cases of antibiotic associated diarrhoea. ${ }^{[3]}$ the incidence of nosocomial $C$. difficile diarrhoea in Canadian hospitals is estimated to range from 38 to 95 cases per 100000 patient-days and from 3.4 to 8.4 cases per 1000 admissions. ${ }^{[4]}$ On 4 June 2003, two outbreaks of a highly virulent strain of this bacterium were reported in Montreal, Quebec, Calgary and Alberta. Sources put the death count to as low as 36 and as high as 89 with around 1,400 cases in 2003 and within the first few months of 2004. ${ }^{[23]}$ On 1 October 2006, C. difficile was said to have killed at least 49 people at hospitals in Leicester, England, over eight months according to National Health Service investigation. Another 29 similar cases were investigated by coroners. ${ }^{[24]}$ On 27 October 2006, nine deaths were attributed to the bacterium in Quebec. ${ }^{[25]}$ In November 2007, the 027 strain spread into several hospitals in southern Finland with 10 deaths out of 115 infected patients reported on 2007-12-14. ${ }^{[26]}$ On 28 May 2011, an outbreak in Ontario had been reported, with 26 fatalities as of 24 July 2011. ${ }^{[27]}$ In 2013, a total of 27 people at one hospital in the south of Sweden were infected with 10 deaths. Five died of the strain $17 .{ }^{[28]}$ As per the recent estimates in 2018 by the Center for Disease Control (CDC), C. difficile infects approximately half a million Americans each year. Among those infected with C. difficile about 29,000 patients had fatal outcomes within a month of the initial diagnosis and 15,000 of these deaths could be directly attributed to the C. difficile infection. Approximately 83,000 of the patients with C. difficile had at least one recurrence and 29,000 died of the initial diagnosis within 30 days. ${ }^{[31,32]}$

\section{BACTERIOLOGY}

Clostridium difficile is a Gram positive, spore-forming rod that causes a spectrum of intestinal diseases extending from relatively mild diarrhoea to toxic mega colon and is a frequent cause of hospital-acquired enteric infection. ${ }^{[7,10]}$ In its spore form the bacterium can survive harsh environments and common sterilization techniques. Spores of $C$. difficile are resistant to high temperatures, ultraviolet light, harsh chemicals, and antibiotics. ${ }^{[3]} C$. difficile colitis has become one of the most important and expensive health-care associated infections. Recurrence rates after 
treatment are high. The role of the immune system in defense against this infection or in the pathogenesis of $C$. difficile-associated disease is poorly defined. ${ }^{[10]}$

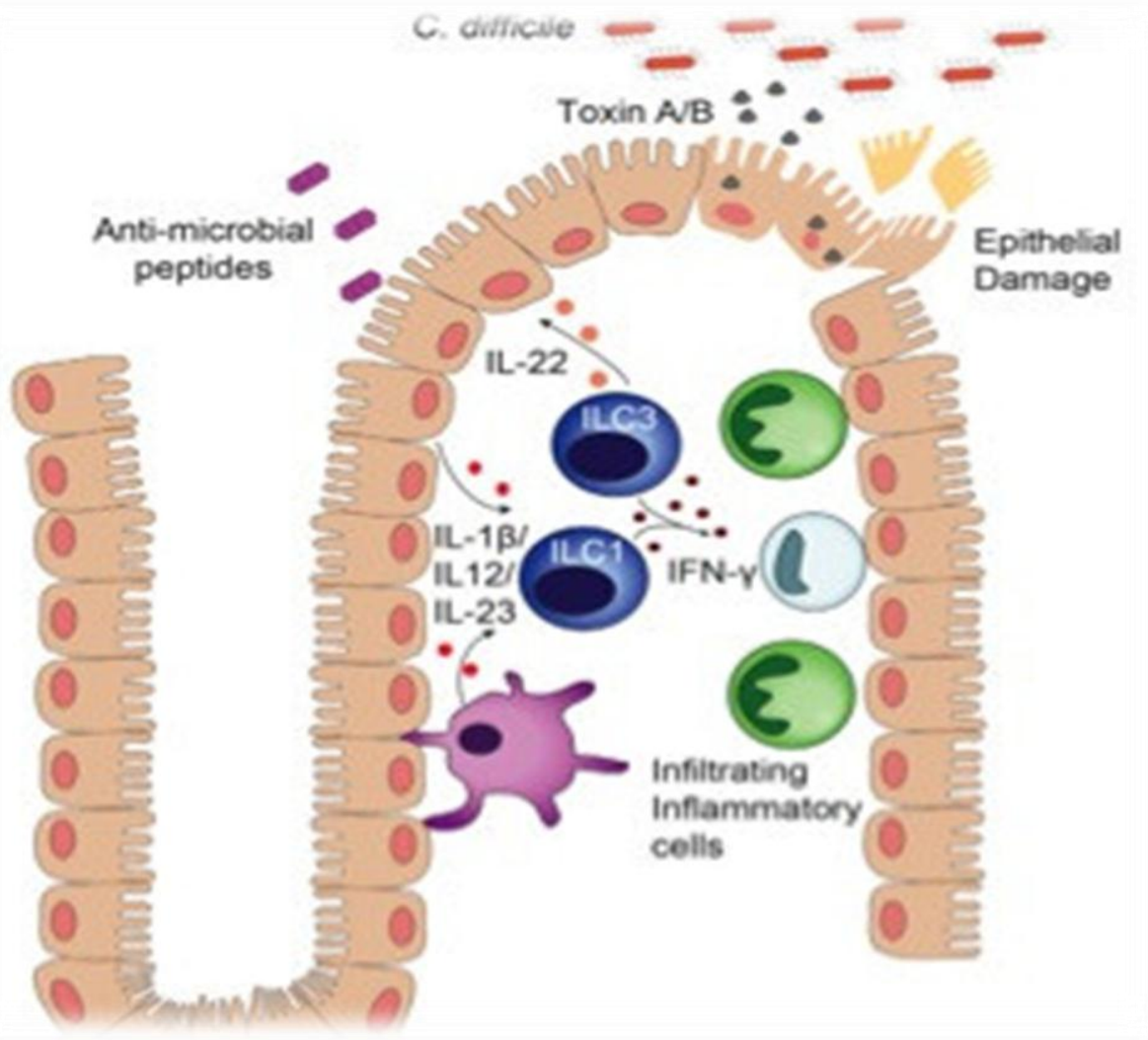

Figure 1: Clostridium difficile produces toxins A and B, which result in a loss of intestinal epithelial integrity ${ }^{[10]}$

\section{PATHOGENESIS}

Pathogenic strains of Clostridium difficile produce two potent exotoxins, enterotoxin (ToxA) and the cytotoxin (ToxB). Clostridium difficile is a Gram-positive, anaerobic, spore-forming, toxinproducing bacillus. ${ }^{[15]} \mathrm{C}$. difficile is the most common cause of nosocomial infections in the United States surpassing methicillin resistant Staphylococcus aureus. ${ }^{[12]}$ Clostridium difficile is acquired through ingestion of spores usually transmitted from other patients through the hands of healthcare personnel or the environment. ${ }^{[11]}$ The spores resist the acidity of the stomach and germinate into the vegetative form in the small intestine. Disruption of normal gut flora typically by exposure to antimicrobials allows C. difficile to proliferate causing a broad spectrum of clinical manifestations that can range from asymptomatic carriage to diarrhoea of varying severity to fulminate colitis and even death. ${ }^{[18]}$ ToxA causes hemorrhagic fluid secretion in the intestinal loop, mucosal inflammation and necrosis of the intestinal tissue (3-5). In contrast to ToxA, ToxB exhibits no overt enterotoxicity. However, both toxins are lethal when injected parenterally into animals. ${ }^{[15]}$ Infection with C. difficile bacteria are responsible for C. difficile 
diarrhoea. Clostridia are anaerobic motile bacteria, ubiquitous in nature and especially prevalent in soil. Under the microscope, they appear as long, irregular (often drumstick or spindle-shaped) cells with a bulge at their terminal ends. ${ }^{[22]}$

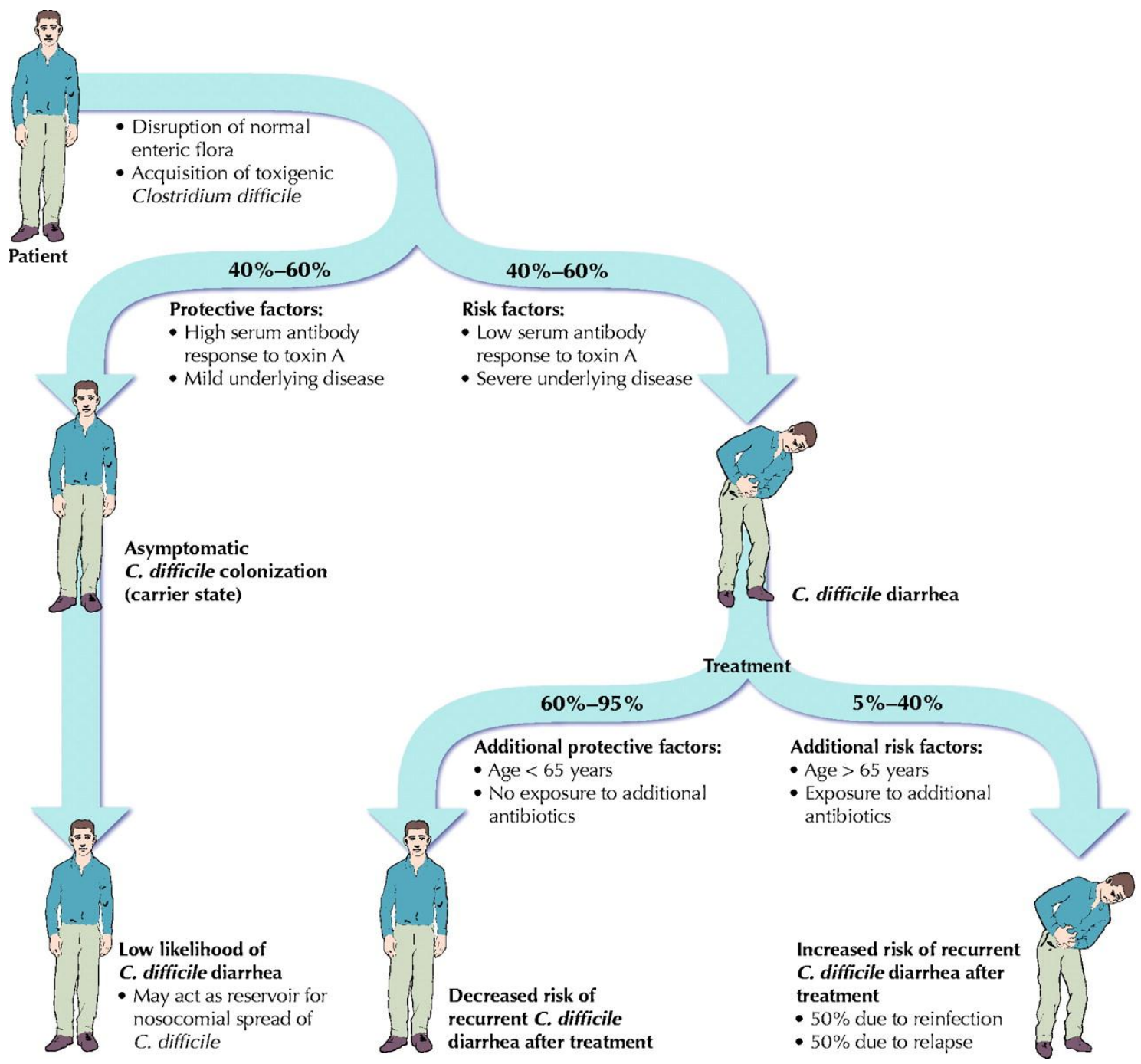

Figure 2: Pathogenesis of Clostridium difficile-associated diarrhoea

\section{EPIDEMIOLOGY}

There has been dramatic change in the epidemiology of Clostridium difficile infection (CDI) since the turn of the 21 st Century noted by a marked increase in incidence and severity occurring at a disproportionately higher frequency in older patients. ${ }^{[19]}$ Rates of CDI have been increasing since 2000, especially in the elderly with a recent hospitalization or residing in long-term care facility (LTCF). Carriage of C. difficile occurs in 5-15\% of healthy adults but may be as high as $84.4 \%$ in newborns and healthy infants and up to $57 \%$ in residents in LTCF. ${ }^{[1]} \mathrm{C}$. difficile diarrhoea is estimated to occur in eight of 100,000 people each year. Among those who are admitted to hospital, it occurs in between four and eight people per 1,000. In 2011, it resulted in 
about half a million infections and 29,000 deaths in the United States. ${ }^{[8]}$ Clostridium difficile ( C. difficile ) is a Gram-positive, spore forming bacterium usually spread by the fecal-oral route. ${ }^{[1]}$ Rates of CDI have been increasing since 2000, especially in the elderly with a recent hospitalization or residing in long-term care facility (LTCF). Carriage of C. difficile occurs in 5$15 \%$ of healthy adults, but may be as high as $84.4 \%$ in newborns and healthy infants, and up to $57 \%$ in residents in LTCF. Transmission in health-care facilities results mostly from environmental surface contamination and hand carriage by staff members and infected patients. $^{[14]}$

\section{Clostridium difficile Toxins}

Most C. difficile strains produce two major toxins, i.e., TcdA and TcdB, generated by the genes TcdA and TcdB within the organism's Pathogenicity loci (PaLoc), while certain C. difficile strains may produce a binary toxin called C. difficile transferase (CDT) closely related to the Clostridium perfringens binary toxin. ${ }^{[12]}$ Additionally, the lethal and hemorrhagic toxins from $\mathrm{C}$. sordelli toxin from $\mathrm{C}$. novyi and the large cytotoxin from $\mathrm{C}$. perfrigens belong to this family. ${ }^{[19,20]}$ However, the incidence of C. difficile infections related to strains only producing CDT is low and the symptoms are moderate. In addition, these strains yield no severe lesions of enteritis in experimental animal models. ${ }^{[12,20]}$

\section{MANAGEMENT ${ }^{[31]}$}

\section{- Management of mild, moderate, and severe CDI}

- If a patient has strong a pre-test suspicion for CDI, empiric therapy for CDI should be considered regardless of the laboratory testing result, as the negative predictive values for CDI are insufficiently high to exclude disease in these patients. (Strong recommendation, moderate-quality evidence).

- Any inciting antimicrobial agent(s) should be discontinued, if possible. (Strong recommendation, high-quality evidence).

- Patients with mild-to-moderate CDI should be treated with metronidazole $500 \mathrm{mg}$ orally three times per day for 10 days. (Strong recommendation, high-quality evidence).

- Patients with severe CDI should be treated with vancomycin $125 \mathrm{mg}$ four times daily for 10 days (Conditional recommendation, moderate-quality evidence).

- Failure to respond to metronidazole therapy within 5-7 days should prompt consideration of a change in therapy to vancomycin at standard dosing.

\section{- Management of severe and complicated CDI}


- Supportive care should be delivered to all patients and includes intravenous fluid resuscitation, electrolyte replacement and pharmacological venous thromboembolism prophylaxis. Furthermore in the absence of ileus or significant abdominal distention, oral or enteral feeding should be continued. (Conditional recommendation, low-quality evidence).

- CT scanning of the abdomen and pelvis is recommended in patients with complicated CDI. (Conditional recommendation, low-quality evidence).

- Vancomycin delivered orally (500 mg four times per day) and per rectum (500 mg in a volume of $500 \mathrm{ml}$ four times a day) plus intravenous metronidazole (500 $\mathrm{mg}$ three times a day) is the treatment of choice for patients with complicated CDI with ileus or toxic colon and significant abdominal distention. (Strong recommendation, low-quality evidence).

\section{- Management of recurrent CDI (RCDI)}

- No effective immunotherapy is currently available. Intravenous immune globulin (IVIG) does not have a role as sole therapy in treatment of RCDI. However, it may be helpful in patients with hypogammaglobulinemia. (Strong recommendation, low-quality evidence).

- The first recurrence of CDI can be treated with the same regimen that was used for the initial episode. If severe, however vancomycin should be used.

- The second recurrence should be treated with a pulsed vancomycin regimen. (Conditional recommendation, low-quality evidence).

- If there is a third recurrence after a pulsed vancomycin regimen, fecal microbiotatransplant (FMT) should be considered. (Conditional recommendation, moderatequality evidence).

- Management of patients with CDI and co-morbid conditions

- In patients who have IBD with severe colitis, simultaneous initiation of empiric therapy directed against CDI and treatment of an IBD fl are may be required while awaiting results of $\mathrm{C}$. difficile testing. (Conditional recommendation, low-quality evidence).

- All patients with IBD hospitalized with a disease are should undergo testing for CDI. (Strong recommendation, high-quality evidence).

\section{RISK FACTORS ${ }^{[9]}$}

\section{Pharmacological risk factors}

Any use of antibiotics (broad and specific)

Any use of proton pump inhibitors 
Any use of histamine 2 receptor antagonists

Anti-ulcer medications (not specific)

Non-steroidal anti-inflammatory drug

Aspirin

Corticosteroids

Use of opiate during the last episode of CDI

\section{Host-related risk factors:}

Age: $\geq 65$ years

Age: additional year or decade

Chronic kidney disease

Diabetes mellitus

Lymphoma or leukemia

Solid cancer or malignancy

Severity of co-morbidity

Inflammatory bowel disease

Congestive heart disease

Chronic obstructive pulmonary disease

Peptic ulcer

Previous diagnosis of CDI

\section{Clinical interventions or characteristics:}

Duration of hospitalization

Nasogastric tube feeding

Stay in intensive treatment unit

Non-surgical GI procedure

Vomiting

History of surgery

Previous gastrointestinal procedure

Serum albumin $<2.5 \mathrm{~g} / \mathrm{dL}$

Lymphopenia

Colonization with vancomycin-resistant enterococci

\section{Treatment}

Treatment of a first episode of recurrent infection with a repeat course of either metronidazole or vancomycin for 10 to 14 days is successful in approximately $50 \%$ of patients. Second and 
subsequent recurrences can be difficult to cure, primarily because of the persistence of spores in the bowel or environment and the inability of the patient to mount an effective immune response to $C$. difficile toxins rather than to antibiotic resistance. Second recurrences can be treated with fidaxomicin or by a vancomycin regimen involving tapered and pulsed dosing. ${ }^{[11,12]}$ Metronidazole and oral vancomycin have been the mainstays of treatment for $C$. difficile infection since the 1970s. For the treatment of severe disease, vancomycin is better than metronidazole but for mild-to-moderate infection the two antibiotics have been considered to be equivalent. However a marked rise in clinical failure associated with metronidazole especially in patients with the BI/NAP1/027 strain, has been seen in the past decade. Previous studies were underpowered to evaluate differences between metronidazole and vancomycin in cases of no severe infection, but recent data suggest an overall superiority of vancomycin. ${ }^{[12]}$

\section{Diagnosis}

The enzyme immunoassay (EIA) for detection of toxins A and B has been the most widely used diagnostic test for CDI because of its rapid turnaround, low cost and simplicity. However EIAs for toxins $\mathrm{A}$ and $\mathrm{B}$ are known to have low sensitivity (60\%-80\%) compared with toxigenic stool culture. ${ }^{[29]}$ detecting toxigenic C. difficile have been developed and currently 5 US Food and Drug Administration-cleared PCR assays are available in the United States. These PCR assays have a short turnaround time and their sensitivities range from $84 \%$ to $94 \%$ compared with toxigenic stool culture, making their use by clinical laboratories very attractive. ${ }^{[30]}$ Only stools from patients with diarrhoea should be tested for Clostridium difficile. (Strong recommendation, high-quality evidence) Nucleic acid amplification tests (NAAT) for C. difficile toxin genes such as PCR are superior to toxins A+ B EIA testing as a standard diagnostic test for CDI. (Strong recommendation, moderate quality evidence) Glutamate dehydrogenase (GDH) screening tests for $\mathrm{C}$ difficile can be used in two- or three-step screening algorithms with subsequent toxin A and B EIA testing, but the sensitivity of such strategies is lower than NAATs. (Strong recommendation, moderate quality evidence). The best standard laboratory test for diagnosis has not been clearly established. For the past 30 years, the two primary reference tests are the C. difficile cytotoxin neutralization assay (CCNA) and toxigenic culture (TC). ${ }^{[31]}$

\section{Future Challenges}

Our knowledge of CDI continues to evolve challenging our understanding of CDI epidemiology. The need for standardized definitions and surveillance methods to detect outbreaks assess disease trends, and facilitate comparison of CDI rates across healthcare institutions was recognized earlier this decade with the increased incidence and severity of CDI. ${ }^{[14]}$ Gaining a better 
understanding of the incidence of CDI and the number of new cases that arise each year is fundamental to understanding the spread of $\mathrm{C}$. difficile within healthcare facilities within the community and from other reservoirs of infection. ${ }^{[17]}$ Lastly, given the shift in risk factors in the community setting, epidemiological studies that only utilize data from the acute care hospital setting under reports a significant proportion of CDI cases skewing severity data and under representing the economic burden of the disease. ${ }^{[16]}$ Another challenge to our understanding of CDI epidemiology is the rapidly changing laboratory diagnostic approaches to CDI. ${ }^{[13]}$ More recently, polymerase chain reaction (PCR) assays, which detect the $\mathrm{C}$. difficile genes involved with toxin production have increased in popularity and offer higher sensitivity and specificity and more rapid turnaround time compared to EIAs. ${ }^{[15]}$ In addition, PCR might diagnose very mild cases of CDI that might resolve without therapy. ${ }^{[2]}$ Patients diagnosed only on the basis of PCR may have fewer complications and less severe disease than patients diagnosed on the basis of toxin detection though this is controversial. ${ }^{[2,5]}$ According to the CDC, C. difficile has become the most common microbial cause of healthcare-associated infections in U.S. hospitals and costs up to $\$ 4.8$ billion each year in excess health care costs for acute care facilities alone. ${ }^{[6]}$

\section{Clinical Practice Guidelines}

The current clinical practice guidelines for CDI in adults updated by the Society of Healthcare Epidemiology of America and the Infectious Diseases Society of America in 2010 state $^{[16]}$ the recent European guidelines on treatment of CDI state that there is insufficient evidence to recommend the addition of probiotics to antibiotics because the studies investigated on probiotics and CDI had significant concerns including small numbers nonrandomized allocations of antibiotics to which the probiotics were added and lack of homogeneity among study groups. ${ }^{[16]}$ To increase comparability between clinical settings use available standardized case definitions for surveillance of healthcare facility-onset (HO) CDI; community-onset, healthcare facilityassociated (CO-HCFA) CDI; and community-associated (CA) CDI (good practice recommendation). ${ }^{[21,16]}$ At a minimum conduct surveillance for $\mathrm{HO}-\mathrm{CDI}$ in all inpatient healthcare facilities to detect elevated rates or outbreaks of CDI within the facility (weak recommendation, low quality of evidence). ${ }^{[21]}$

\section{CONCLUSION}

Clostridium difficile is a Gram positive, spore-forming rod that causes a spectrum of intestinal diseases extending from relatively mild diarrhoea to toxic mega colon and is a frequent cause of hospital-acquired enteric infection. We suggest treatment of patients be stratified depending on 
whether they have mild-to-moderate, severe or complicated disease. The current review highlights the current knowledge on $C$. difficile how to produce infection in human, transmission, guidelines, Epidemiology, risk factors, diagnosis and future challenges. It also provides a detailed report on treatment and management of Clostridium Difficile Infection (CDI).

\section{AKNOWLEDGMENTS}

We are thankful to Dr. Shirsat M. K. and all authors for their supports, co-operation and guidance in searching various articles and journals for completion of this review article and also thank to Loknete Shree Dadapatil Pharate College of Pharmacy, Mandavgan Pharata, Pune.

\section{REFERENCES}

1. Hall I, O'Toole E. Intestinal flora in newborn infants with a description of a new pathogenic anaerobe, Bacillus difficilis. Am J Dis Child. 1935; 49:390.

2. Longtin $\mathrm{Y}$, Trottier S, Brochu G, et al. Impact of the type of diagnostic assay on Clostridium difficile infection and complication rates in a mandatory reporting program. Clin Infect Dis. 2013; 56:67-73.

3. Latisha Heinlen, et al. Clostridium difficile Infection. Am J Med Sci. 2010 Sep; 340(3): $247-252$.

4. Hyland M, et al. Canadian Hospital Epidemiology Committee, Canadian Nosocomial Infection Surveillance Program (Health Canada). Can J Infect Dis. 2001 Mar; 12(2):81-8

5. Wilcox MH. Overcoming barriers to effective recognition and diagnosis of Clostridium difficile infection. Clin Microbiol Infect. 2012; 18(6):13-20.

6. Bartlett JG. Review Clostridium difficile: history of its role as an enteric pathogen and the current state of knowledge about the organism. Clin Infect Dis. 1994 May; 18(4):S265-72.

7. Johnson S, Adelmann A, Clabots CR, Peterson LR, Gerding. Recurrences of Clostridium difficile diarrhoea not caused by the original infecting organism. DNJ Infect Dis. 1989 Feb; 159(2):340-3.

8. https://en.wikipedia.org/wiki/Clostridium_difficile_infection

9. https://www.ncbi.nlm.nih.gov/pmc/articles/PMC5460399/

10. https://www.mskcc.org/research-areas/labs/eric-pamer/clostridium-difficile-infection.

11. Susan M. Poutanen et al. Clostridium difficile-associated diarrhoea in adults. CMAJ July 06, 2004; 171(1) 51-58. https://doi.org/10.1503/cmaj.1031189.

12. https://blogs.nejm.org/now/index.php/clostridium-difficile-infection/2015/04/17/ 
13. Freeman J, Bauer MP, Baines SD, et al. The changing epidemiology of Clostridium difficile infections. Clin Microbiol Rev. PubMed. 2010; 23:529-549.

14. Lessa FC, Gould CV, McDonald LC. Current status of Clostridium difficile infection epidemiology. Clin Infect Dis. PubMed. 2012; 55(12):S65-S70.

15. Surawicz CM, Brandt LJ, Binion DG, et al. Guidelines for Diagnosis, Treatment and Prevention of Clostridium difficile Infections. Am J Gastroenterol. 2013 Feb 26.

16. Khanna S, Pardi DS, Aronson SL, et al. The epidemiology of community-acquired Clostridium difficile infection: a population-based study. Am J Gastroenterol. PubMed. 2012; 107:89-95.

17. Fitzpatrick F, Barbut F. Breaking the cycle of recurrent Clostridium difficile infections. Clin Microbiol Infect. PubMed. 2012; 18(6):2-4.

18. Kuijper EJ, Coignard B, Tüll P; ESCMID Study Group for Clostridium difficile; EU Member States; European Centre for Disease Prevention and Control. Emergence of Clostridium difficile-associated disease in North America and Europe. Clin Microbiol Infect 2006; 12(6):2-18.

19. Jank, T.; Giesemann, T.; Aktories, K. Rho-glucosylating Clostridium difficile toxins A and B: New insights into structure and function. Glycobiology [CrossRef] [PubMed]. 2007, 17, 15-22.

20. Pruitt, R.N.; Lacy, D.B. Toward a structural understanding of Clostridium difficile toxins A and B. Front. Cell. Infect. Microbiol. [CrossRef] [PubMed]. 2012, 2, 28.

21. L Clifford McDonald, et al. Clinical Infectious Diseases, 66(7), 19 March 2018, Pages e1-e48.

22. Ryan KJ, Ray CG Sherris Medical Microbiology McGraw Hill. 4th ed. (2004). pp. 3224.

23. https://en.wikipedia.org/wiki/Clostridium_difficile_infection\#cite_note-Sherris-12]

24. Trust confirms 49 superbug deaths". BBC News Online. 1 October 2006. Archived from the original on 22 March 2007.

25. https://www.ncbi.nlm.nih.gov/pmc/articles/PMC1828183/

26. https://emedicine.medscape.com/article/186458-overview

27. https://www.cbc.ca/news/health/clostridium-difficile-faqs-1.730082

28. http://www.acmicrob.com/microbiology/clostridium-difficile-a-neglected-but-emergingpathogen-in-india.php?aid=6501 
29. Fernanda C. Lessa, Carolyn V. Gould, and L. Clifford McDonald. C. difficile Epidemiology. Clinical Infectious Diseases 2012;55(2):S65-70.

30. Eastwood K, Else P, Charlett A, Wilcox M. Clostridium difficile toxin detection assays, a real time PCR assay for C. difficile J Clin Microbiol 2009; 47:3211-7.

31. Christina M. Surawicz, et al, Guidelines for Diagnosis, Treatment and Prevention of Clostridium difficile Infections. Am J Gastroenterol 2013; 108:478-498.

32. Pradeep Kumar Mada, Mohammed U. Alam, Clostridium Difficile StatPearls Publishing. June 5, 2018. 